\title{
Signal Processing Methods for Improvement of Environmental Perception of Persons with Deafblindness
}

\author{
Parivash Ranjbar \\ Audiological Research Centre, Örebro University Hospital, Örebro, Sweden \\ School of Health and Medical Sciences, Örebro University, Örebro, Sweden \\ Campus Alfred Nobel, Örebro University, Karlskoga, Sweden \\ Parivash.ranjbar@oru.se, Parivash.ranjbar@orebroll.se
}

Keywords: Haptic; Vibrotactile; Signal processing; Deafblindness; Environmental perception; Deaf; Monitor.

\begin{abstract}
Environmental perception is a functional area that is severely limited in persons with deafblindness (DB) who belong a category of people with severe disabilities. Monitor is a vibratory aid developed with the aim to improve environmental perception of persons with DB. The aid consists of a mobile phone with an application connected to a microphone and vibrator. Monitor picks up the sounds produced by events by microphone, processes the sound using an algorithm programmed as an application in the mobile phone and then presents the signal via the vibrator to the persons with DB to be sensed and interpreted. In previous laboratory studies, four algorithms (AM, AMMC, TR, and TRHA) were developed based on modulating, and transposing principles. The algorithms were tested by persons with normal hearing/hearing impairment and selected as good candidates to improve vibratory identification of environmental sounds. In this on-going the algorithms are tested by 13 persons with congenital D and five persons with DB using Monitor in a realistic environment, living room, kitchen or office. Forty five recorded environmental sounds were used as test stimuli.

The subjects tested the algorithms two times, Test and Retest each including a test session initiated by a training session. The four algorithms were tested in four days at Test and four days at Retest in total eight test days. Each test day began with a training session where a sound was presented as vibrations to be sensed by the person with the aim to remember its pattern and identity. The 45 sounds were grouped in four groups where an specific algorithm was chosen to process an specific sound group in a specific day. At the test session a sound was presented and the person was given 5 randomly chosen sound alternatives to choose the one as represented sound. The algorithms were different for different sound groups during four different test days so all algorithms were used to process all sounds. The algorithms were tested a second time, Retest, in same way as in Test.

The mean value of identification of environmental sounds varied between $74.6 \%$ and $84.0 \%$ at Test and between $86.9 \%$ and $90.4 \%$ at Retest. The identification results at Retest were significantly improved $(\mathrm{p}<0.01)$ for all algorithms after a relatively short time of training indicating a good learning effect. At Test the algorithm AM was significantly better than the algorithms AMMC and TRHA $(\mathrm{p}<0.01)$ and the algorithm TR was better than TRHA $(\mathrm{p}<0.01)$.

The algorithms AM, AMMC, and TR were selected as good candidates to be implemented in the Monitor to improve environmental perception.
\end{abstract}




\section{Introduction}

People with deafblindness (DB) are the category with severe disabilities at the three functional areas mobility, Communication and Environmental perception. For mobility, they have the white cane or guide dog. For communication, they use e.g. email, Braille, or tactile sign language,... For the environmental perception, which is the focus of this study there are no specific technical aids.

Environmental perception is necessary to feel involved in on-going events, the sequence of events, in the long and short term. Perception of the environment can be divided into five levels: the event is detected, located, identified, interpreted and a decision to reaction (or passivity) is made, thereby an influence on the event is imposed [1]. People with normal hearing and vision can detect and locate events because they can see what is happening and hear the sound generated by the events. How the person identifies and interprets the events depends on the memory s(he) has of the events and the context in which they occur (See Fig. 1). The figure can be adapted even in robotic context where different sensors do the first step, detection, and an artificial intelligence method does the remaining steps, location, interpretation, and decision to reaction.

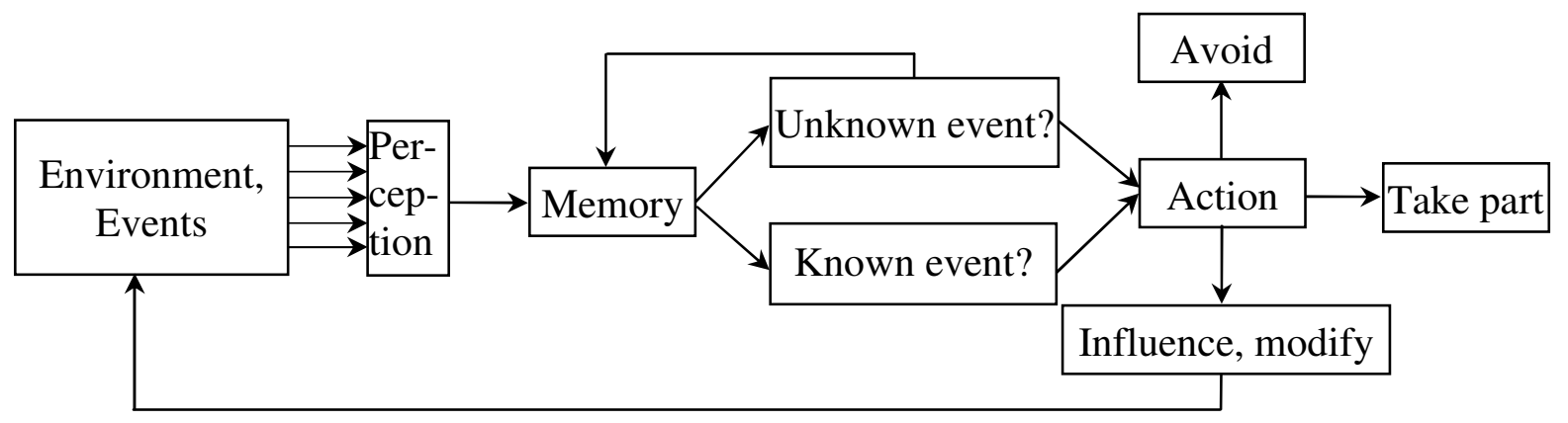

Fig. 1: The relationship between the human surrounding, events, and his/her actions.

Persons with DB experience environmental perception as a major problem because the vision and hearing senses cannot compensate each other as in persons with deafness (D) or blindness. They do not notice an approaching person until the person touches them or the person with DB perceives the situation by smell when (s)he at a short distance senses the person's breath or perfume. The limited ability of persons with DB to get information about on-going events makes it difficult to have an appropriate forward planning and control (adapt to and influence).

Persons with severe hearing impairment (HI) with minimal residual hearing who do not benefit from their hearing aids (HA) to understand speech, often use their HA to hear environmental sounds [2]. These persons may miss some environmental sounds because a HA typically is programmed for the speech and not for the environmental sounds that often has a different spectrum than speech. For persons with no residual hearing, the skin senses become most important and they perceive their environment through information via vibrations from events producing sounds. The ability of skin senses to perceive the sounds in the sensitivity range of hearing $(20-20000 \mathrm{~Hz})$ is limited and the sounds should be adapted to the sensitivity range of the skin $(<1000 \mathrm{~Hz})$ [3]. The persons with DB have shown interest in a vibrotactile aid informing them about ongoing events [4]. The vibrotactile aids e.g. Minivib and Tactaid VII which are designed for speech, convey certain environmental information but miss most of the environmental sounds since they have different spectrum than the speech $[5,6]$. In this on-going project the vibrotactile aid Monitor, an aid to improve environmental perception has been developed to help persons with severe HI/D and/or DB to detect that something is happening and identify what is happening [1,7]. The aid, Monitor, uses the sense of touch as input channel to inform and is programmed for environmental sounds and not for speech $[1,7]$.

In series of studies a non-portable version of the vibrotactile aid Monitor was evaluated in different laboratory and field studies. The studies resulted in development of four algorithms (AM, AMMC, TR and TRHA) for vibratory identification of environmental sounds which were chosen as good candidates to be implemented in a portable vibrotactile aid [1]. A portable version of Monitor was developed consisting of: a mobile phone containing an application to process sound, an 
external microphone to pick up sounds and a vibrator to present the processed sound [7]. Four persons with DB having Usher Syndrome I, (US I, who are born deaf and lose successive their vision) evaluated the Monitor in a field study. The study was initiated by an experiment with the aim, using the Monitor and persons with congenital D/DB test and compare the four algorithms and select the algorithm(s) resulting in highest scores of vibratory identification of environmental sounds. The selected algorithm for a specific person with DB was supposed to be implemented as an application in the Monitor and used by the person with the aim to test and evaluate the Monitor in a field study after long time training. In this report the experiment initiating the field study performed by persons with $\mathrm{D} / \mathrm{DB}$ is presented.

\section{Purpose}

The purpose of the present study is: using the vibrotactile aid Monitor and persons with congenital D/DB test and compare the four algorithms and select the algorithm(s) leading to in highest results of vibratory identification of environmental sounds.

\section{Method}

Subjects: Eighteen volunteers $(13 \mathrm{~F} / 5 \mathrm{M})$ with congenital D (hearing thresholds $<90 \mathrm{~dB} \mathrm{HL}$ ) between 20-55 years of age (median 35.5 years) participated in the tests. Six of participants (Ps) were females with Usher Syndrome I [8] where five of them had small remaining vision and one had only light perception. The Ps were recruited from Örebro County by sending email via the Association of the Swedish Deafblind (FSDB, Förbundet Sveriges Dövblinda). The Ps could suggest their friends or colleges who could possibly meet the criterions and were willing to participate in the tests. Written information about the test, procedure, criterions of Ps and contact information for the project manager was sent to those who met the requirements and had notified their interest to the project manager. Information about Ps hearing and vision was collected after the test leader had received Ps consent. All communication occurred via email or sign language.

Monitor: Monitor, the vibrotactile aid used in the experiments consists of: a mobile phone (HTC, based on Android) containing an application to process sound, an external microphone (Phonac MM8) for detecting sounds and can be placed on any body part where the $\mathrm{P}$ wishes (so that it is neither hidden nor in the way) and a vibrator (C2-Tactor), which can be placed on the body part where $\mathrm{P}$ has high vibration sensitivity and feel comfortable [7]. The microphone and the vibrator are connected to a mobile phone via the head sett plug. Between mobile phone and the vibrator there is an amplifier (Wowpotas). Monitor picks up the sounds produced by events by microphone, processes the sound using an algorithm programmed as an application in the mobile phone and then presents the signal via the vibrator to the persons with DB to be sensed.

Stimuli: 45 recorded environmental sounds were used as stimuli. These sounds were chosen as representative and important sounds to be informed about, by a panel of 11 persons with normal hearing and vision in addition to some persons with DB [9]. These sounds have been used as stimuli also in previous studies [9-11].

Applications: The application loaded in the mobile phone, Monitor, was programmed with one of the four algorithms (AM, AMMC, TR and TRHA) shown in the Table I. These algorithms were tested by Ps with D and/ or normal hearing in previous laboratory studies [1] and selected as good candidates to be implemented in a vibratory aid for improvement of environmental perception. For more detailed descriptions of the algorithms see the studies $[1,9,10]$. 
Table I. The four algorithms used to process the environmental sounds used in this experiment.

\begin{tabular}{ll}
\hline Abbreviation & Description \\
AM & Amplitude Modulation of a $250 \mathrm{~Hz}$ carrier wave with the envelope of in signal \\
AMMC & Amplitude Modulation of the carrier waves $43,86,129,201,244$ and $301 \mathrm{~Hz}$ with \\
the envelope of the output from the Multiple Channels filtered in the different cut \\
off frequencies $120-240,240-480,480-960,960-1920,1920-3840$ and $3840-$ \\
$5500 \mathrm{~Hz}$, respectively. The modulated signals were multiplied by the factors $4,3.5$, \\
\\
2, $1.5,1$, and 1.5 respectively and added to build the output signal. \\
TRansposing data in the range $0-5500 \mathrm{~Hz}$ to the range $0-290 \mathrm{~Hz}$ by passing the in \\
signal through four filters, one low pass filter at cutoff frequency $100 \mathrm{~Hz}$ and three \\
band pass filters with cutoff frequencies $100-1200,1200-2400,2400-5500 \mathrm{~Hz}$ \\
respectively. The envelope of the outputs from the band pass filters were used to \\
amplitude modulate the carrier waves 158,215 and $290 \mathrm{~Hz}$ respectively. The total \\
output was obtained by adding the output from low pass filter and the three \\
modulated signals. \\
TRansposing the 10 frequency components with Highest Amplitude in the range \\
TRHA $5500 \mathrm{~Hz}$ to the range $52-470 \mathrm{~Hz}$. The 10 selected frequencies got the new \\
positions $32,52,157,118,222,280,314,420,440$, and $470 \mathrm{~Hz}$ where they had \\
same order as in the original sound.
\end{tabular}

\section{Procedure}

The experiment consisted of two parts: Test and Retest which were performed in total in eight days (four days for each part). The tests performed in each day were initiated by a training session and followed by a test session.

The Ps were sitting in a relaxed manner in the living room, kitchen or office and placing the vibrator of Monitor under a sweatband to sense the vibrations via ankle, the palmar side of the hand. The microphone of Monitor was clamped in front of a loudspeaker connected to a computer and had same position during both training and test sessions. The test leader had access to a sound list where the sounds were grouped in four different to be processed by a specific algorithm e.g. AM (1-11), AMMC (12-23), TR (24-35), and TRHA (36-45) The order of algorithms which was unknown for the Ps was randomly chosen for each sound group at the first test day. In the following three test days their order were changed as in permutation, continuously in a way that all four algorithms were used to process all 45 sounds during the four different test days. The stimuli, 45 recorded environmental sounds, were played one by one through the loudspeaker, detected, processed by a chosen algorithm and presented via vibrator to be sensed and identified by the $\mathrm{P}$.

At each test day, first the $\mathrm{P}$ was trained by sensing each sound (presented from number 1 to 45 ) as vibrations processed by the specific pre decided algorithm in the list and getting information about its identity which was sometimes new for the Ps since they were born deaf and no one had informed them about it. The Ps were encouraged to focus and remember the pattern of the vibrations specific to the presented sound. After training, at the test session the sounds were processed with same algorithm as in the training part with the difference that the sounds in each group were randomly presented. For each presented sound, the Ps got five randomly chosen sound alternatives which were the same alternatives for the same presented sound during all four days. The order of algorithms and the sound alternatives were different for different Ps. The alternatives were presented as text for the Ps with D and normal vision and sign language for the persons with DB who had to remember the sound alternatives. The P could choose one of the five alternatives as the presented sound. The experiment continued from one day to another day in the same way where the algorithms were changed for each sound group without any feedback. Professional interpreters were used when persons with DB performed the experiments which could take between 3 and 4 hours inclusive break. The test was interrupt for a break when the $\mathrm{P}$ och the interpreters asked. The number of interpreters could vary between two and four according to Swedish rules. 
The Test part was repeated as Retest. At Retest no professional interpreters were used since the Ps with DB became stressed when several persons were waiting for a response from the P. The test leader communicated with the Ps using sign language. The Ps got the five sound alternatives as a text printed with a font style and text size adapted to the Ps visually ability. For P2 (no residual vision) the sound alternatives were presented as Braille via her computer. All Ps were satisfied with this new communication way and preferred tests without interpreters.

\section{Results}

The identification scores were calculated by summing up the number of correctly identified sounds by each P testing each algorithm (AM, AMMC, TR and TRHA) at Test and Retest. The obtained identification scores are shown in the Fig. 2.

The mean value of identification results for each algorithm was relatively high (better than the guessing level 20\%) both in Test (74.6\%-84.0\%) and Retest (86.9\%-90.4\%).

At Test, the mean values of the results were for $\mathrm{AM}=84.0 \%, \mathrm{AMMC}=79.7 \%, \mathrm{TR}=81.4 \%$ and for TRHA $=74.6 \%$. The corresponding values at Retest were for $\mathrm{AM}=90.4 \%, \mathrm{AMMC}=90.0 \%$, $\mathrm{TR}=89.3 \%$ and TRHA $=86.9 \%$. The results at Test and Retest were evaluated using two-way ANOVA which showed significantly improvement of the results at Retest $(p<0.01)$ for all algorithms. At Test the algorithm AM was significantly better than the algorithms AMMC and TRHA $(\mathrm{p}<0.01)$ and the algorithm TR was better than TRHA $(\mathrm{p}<0.01)$. At Retest there were no significant differences between the algorithms except for the algorithm AMMC which was significantly better than the algorithms TR and TRHA.
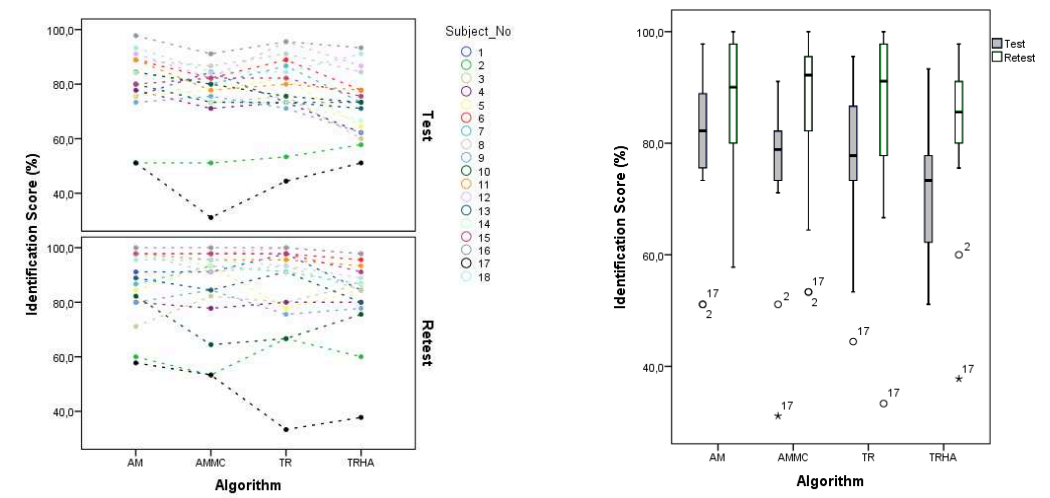

Fig. 2 The results of identification of environmental sounds processed using four different algorithms by 18 subjects during four days two times, Test and Retest where $100 \%=45$ items correct. Left: The individual identification scores. Right The results: median; 25 and 75 percentiles; minimum and maximum values. The subjects 2 and 17 were outliners.

At the Retest the Ps were more certain and their response time was much shorter in the Test. They responded often before the whole stimulus was presented. The test time of some Ps was reduced to shorter than one hour.

The Ps number 2 and 17 were the two outliners with low identification scores. The P2 had only light perception and received the sound alternatives tactually or via Braile and had difficult to remember all. The P17 was often tired and was sleepy but still wanted to continue the tests. 


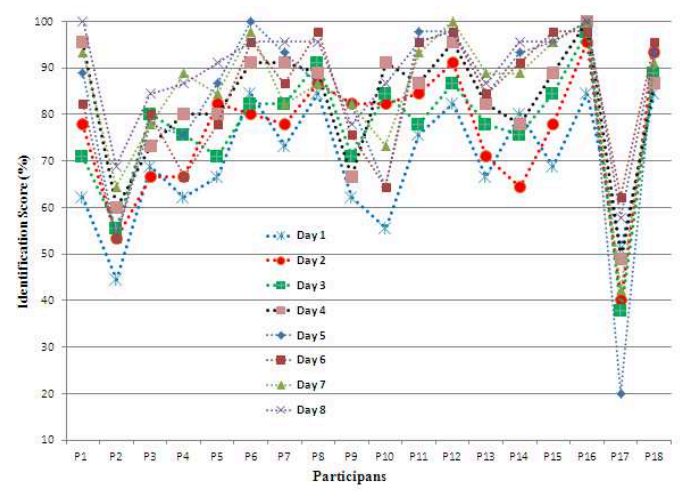

Fig. 3 The results of identification of environmental sounds during eight test days, Test (Day 1-Day 4) and Retest (Day 5-Day 8).

There were a positive effect of training and learning. The mean value of identification results of the Ps at one test day was increased from Day 1 to Day 8 as follow: $69.9 \%, 76.3 \%, 77.3 \%, 82.5 \%$, $82.8 \%, 85.1 \%$, and $89.1 \%$ respectively. The results of P17 (outliner at Fig. 2 left) were increased from $(20 \%)$ at the Day 1 to $61 \%$. The increment was lower at the Retest since the Ps had already got their maximum level e.g. P16 could identify $100 \%$ correctly already at Day 4 . In some cases the Ps could identify later but they were not allowed to go back and change the responses. The results of each algorithm at Test and Retest were added and compared using paired T-test which showed that the algorithm AM, AMM, and TR were significantly $(\mathrm{p}<0.05)$ better than algorithm TRHA, and the algorithm AM was also significantly $(\mathrm{p}<0.05)$ better than the algorithm AMMC.

The Ps experienced the sounds processed by the algorithm TRHA as weak and increased the intensity level at the training session. At the test session, when testing algorithm TRHA the test leader increased the intensity to same level as the Ps had at the training session.

\section{Discussion}

All the Ps are born deaf and had same conditions in terms of hearing ability. On the other hand, older people have had more time to practice to receive information via natural low frequency sounds and vibrations than the younger ones. Each test session was initiated by a training session and thereby reduced differences in vibratory experience level. The identification scores of the Ps number 2 and 17 were lower than results of the remaining Ps which can be explained by their cognitive abilities. They had however, also benefit from Monitor since their results were better than the guessing level (20\%).

The tool had the same structure for all Ps, the vibrator could be placed where the Ps are sensitive to vibrations and feel comfortable. All Ps chose to have it on their palm under a sweatband.

The Monitor was used and tested in the Ps' residence area home which was different for each $\mathrm{P}$ and thereby different reverberation time. On the other hand they were tested often at the kitchen and the microphone was close to the amplifier. The conditions were the same for each $\mathrm{P}$ and different algorithms.

The algorithms were modified in some extension but they were developed on the same principles as the algorithms in the previous study namely modulation and transposition where they were selected as good candidates to process environmental sounds. The Ps experienced the sounds processed using the algorithm TRHA as weak which can partly depend on its spectrum which was between 32 and $470 \mathrm{~Hz}$. The vibrator had a non-linear spectrum with logarithmic attenuation at the frequencies above $100 \mathrm{~Hz}$. The signal should be adapted and compensated regarding the vibrators spectrum. All algorithms should also be adapted to the vibratory sensitivity thresholds of the skin. In the previous study the algorithms had two alternatives (adapted and un-adapted) which did not show any significant differences.

At the first test day the Ps were inexperienced and the vibrations, algorithms, sounds... were new for the Ps. To minimize the effect of training on the relation between day, all four algorithms were 
tested in a random order at the first day. The order of the algorithms in the three remaining test days were changed continuously so that each algorithm could have the different orders one, two, three or four in whole experiment. The response alternatives for each presented sound were chosen randomly for different Ps but they were the same for each $\mathrm{P}$ at the four test days. The number of response alternatives was five. The alternatives more than five would be difficult for the Ps to remember since the alternatives were presented in sign language which required an optimal functioning cognitive capacity to remember the alternatives to select one. At the Retest the alternatives were presented as text (they could see one two or three alphabets or one word at the time) or Braile which also required functioning cognition.

All four algorithms had almost equally high mean value of identification scores (between $86.9 \%$ and $90.4 \%$ at Retest) after short times training. The algorithm TRHA should be improved by adapting its spectrum also to the frequency range of the vibrator.

Finally it can be concluded that the algorithms AM, AMMC, and TR are similarly good candidates to be implemented in the vibratory aid Monitor. The algorithms can be analyzed further regarding different sounds with the aim to modify the algorithms or develop different algorithms for sounds having different spectrum. A possibly preferable algorithm would be one getting benefit from all four algorithms where as in robots it choses automatically an appropriate algorithm after analyzing the input signal.

\section{Reference}

[1] Ranjbar, P., Sensing the Environment: Development of Monitoring Aids for Persons with Profound Deafness or Deafblindness, in School of Science and Technology2009, Örebro Universitet: Örebro p. 188.

[2] Sundström, B. and E. Borg, Gravt hörselskadade och vuxendövas nytta och/eller besvär med hörapparat, 2004, Ahlséns Forskningsinstitut: Örebro. p. 86.

[3] Borg, E., Cutaneous senses for detection and localization of environmental sound sources: a review and tutorial. Scand Audiol, 1997. 26(4): p. 195-206.

[4] Borg, E., et al. Monitoring environmental events: problems, strategies and sensory compensation. in ISAC'00 Conference. 2000. Exeter.

[5] Reed, C.M. and L.A. Delhorne, The reception of environmental sounds through wearable tactual aids. Ear and Hearing, 2003. 24(6): p. 528-538.

[6] Spens, K.-E. and G. Plant, A tactual "hearing" aid for the deaf. STL-QPSR, 1983. 24(1): p. 5256.

[7] Ranjbar, P. and I. Stenstrom, Monitor, a vibrotactile aid for environmental perception: a field evaluation by four people with severe hearing and vision impairment. ScientificWorldJournal, 2013. 2013: p. 206734.

[8] Sadeghi, M., Usher syndrome. Prevalence and phenotype - genotype correlations, in Department of Audiology, The Sahlgrenska Academy2005, Göteborg University: Göteborg. p. 191.

[9] Ranjbar, P., et al., Auditive identification of signal-processed environmental sounds: Monitoring the environment. International Journal of Audiology, 2008. 47(12): p. 724 - 736

[10] Ranjbar, P., E. Borg, and D. Stranneby, Vibrotactile identification of signal-processed sounds from environmental events. Journal of Rehabilitation Research and Development, 2009. 46(3).

[11] Ranjbar, P., Vibrotactile identification of signal-processed sounds from environmental events presented by a portable vibrator: A laboratory study. Iranian Rehabilitation Journal, 2008. 6(7 \& 8): p. 24-38. 